\title{
Efeito do resfriamento evaporativo e do balanço eletrolítico sobre a lactação de porcas em condições de verão tropical
}

\author{
[Effect of evaporative cooling and electrolyte balance on lactating sows \\ in tropical summer conditions] \\ E. Justino ${ }^{1}$, I.A. Nääs ${ }^{1}$, T.M.R. Carvalho ${ }^{1}$, D.A. Salgado ${ }^{2}$ \\ ${ }^{1}$ Universidade Estadual de Campinas - UNICAMP - Campinas, SP \\ ${ }^{2}$ Pontifícia Universidade Católica de Campinas - PUC - Campinas, SP
}

\section{RESUMO}

O experimento foi realizado em uma granja comercial com 72 fêmeas suínas da genética DanBred ${ }^{\circledR}$ e objetivou avaliar os efeitos do resfriamento evaporativo e do balanço eletrolítico sobre o desempenho e os parâmetros de termorregulação de porcas lactantes no verão. O delineamento experimental foi blocos ao acaso com quatro tratamentos em disposição fatorial 2 x 2 e 18 repetições por tratamento. Os tratamentos foram dois sistemas de ventilação (ventilação natural ou resfriamento evaporativo) e duas dietas (balanço eletrolítico de $175 \mathrm{mEqkg}^{-1}$ ou balanço eletrolítico alto de $275 \mathrm{mEqkg}^{-1}$ ). Os valores médios encontrados para temperatura ambiente e umidade relativa do ar foram de $24,8^{\circ} \mathrm{C}$ e $64,5 \%$, respectivamente, sendo obtidos valores diários de $31,6^{\circ} \mathrm{C}$ para a temperatura ambiente máxima e de $19,8^{\circ} \mathrm{C}$ para a temperatura ambiente mínima. Não houve efeito $(\mathrm{P}>0,05)$ significativo do resfriamento evaporativo e do balanço eletrolítico sobre o consumo de ração, a condição corporal e o intervalo desmame-estro das porcas. O sistema de resfriamento evaporativo proporcionou redução $(\mathrm{P}<0,05)$ na frequência respiratória e temperatura superficial mensuradas nos períodos da manhã e da tarde e na temperatura retal à tarde e aumento $(\mathrm{P}<0,10)$ no peso dos leitões ao desmame. A dieta com balanço eletrolítico alto não influenciou essas variáveis. Concluiu-se que a utilização do sistema de resfriamento evaporativo contribuiu para reduzir os efeitos do calor sobre as variáveis relacionadas à termorregulação das porcas em lactação durante o verão e proporcionou aumento no peso dos leitões ao desmame. Em dieta com níveis nutricionais específicos para climas quentes, o balanço eletrolítico alto não minimizou os efeitos do estresse calórico.

Palavras-chave: desempenho, dieta, suínos, termorregulação, ventilação

\begin{abstract}
The field trial was conducted in a commercial farm with 72 sows from DanBred ${ }^{\circledR}$ genetics and aimed to evaluate the effects of the evaporative cooling and electrolyte balance on the performance and thermoregulatory parameters of lactating sows during summer. The experimental design was randomized blocks with four treatments in a factorial arrangement $2 \times 2$ and 18 replicates per treatment. Treatments were two ventilation systems (natural ventilation or evaporative cooling) and two diets (electrolyte balance of $175 \mathrm{mEqkg}{ }^{-1}$ or high electrolyte balance of $275 \mathrm{mEqkg}{ }^{-1}$ ). The average values for temperature and relative humidity were $24.8^{\circ} \mathrm{C}$ and $64.5 \%$, respectively, being obtained daily values of $31.6^{\circ} \mathrm{C}$ for the maximum temperature and of $19.8^{\circ} \mathrm{C}$ for the minimum temperature. There was no effect $(P>0.05)$ of evaporative cooling and electrolyte balance on feed intake, body condition and weaning-to-estrus interval of sows. The evaporative cooling caused a significant reduction $(P<0.05)$ in respiratory rate and surface temperature measurements in the morning and afternoon and evening rectal temperature and increase $(P<0.1)$ in the weight of piglets at weaning. The dietary high electrolyte balance did not change these variables. It was concluded that the use of the evaporative cooling system helped to reduce the effects of heat on the variables related to body thermoregulation during the summer and provided an increase in weaning piglet weight. The utilization of a diet with specific nutrient levels for hot climates, the high electrolyte balance did not reduce the effects of heat stress in sows.
\end{abstract}

Keywords: performance, diet, pigs, thermoregulation, ventilation

Recebido em 6 de fevereiro de 2013

Aceito em 10 de junho de 2014

E-mail: eliene.justino@gmail.com 


\section{INTRODUÇÃO}

Durante o período de lactação, as fêmeas devem consumir níveis adequados de ração para mantença e produção de leite, já que o baixo consumo resulta em mobilização das reservas corporais, condição corporal inadequada, falhas reprodutivas e redução no tempo de vida útil das fêmeas no rebanho (Bloemhof et al., 2008). A otimização do consumo de nutrientes é uma forma de evitar problemas reprodutivos póslactação devido à perda excessiva de peso das porcas durante a lactação (Lesskiu et al., 2011). Porém, em condições de temperatura ambiente elevada, as fêmeas suínas reduzem a ingestão de alimentos, a fim de minimizar a produção de calor. Segundo Kemp et al., (2011) os efeitos negativos da temperatura elevada sobre as porcas são mais prejudiciais durante os períodos médio e final da lactação, quando a produção de leite e o consumo de ração são maiores.

O aumento da frequência respiratória é um mecanismo utilizado para eliminar o calor corporal; no entanto, ocasiona uma mudança no equilíbrio ácido-base (Savaris, 2008) e o aparecimento da alcalose respiratória. Um dos métodos usados para restabelecer a homeostase (equilíbrio interno) é a manipulação do balanço eletrolítico da dieta (Patience, 1990). Outra medida para minimizar as perdas decorrentes do estresse calórico é a utilização de ventilação forçada (Teixeira et al., 2004), resfriamento do piso (Silva et al., 2006; Lima et al., 2011) ou resfriamento evaporativo localizado na região dorsal, sobre a cabeça da porca, com o objetivo de aumentar a perda de calor para o ambiente e reduzir a temperatura superficial da pele (Aarnink et al., 2006; Panagakis e Axaopoulos, 2006; Martin, 2012).

Portanto, o objetivo deste trabalho foi avaliar os efeitos do resfriamento evaporativo e do uso da dieta com balanço eletrolítico alto sobre o desempenho e os parâmetros de termorregulação de porcas em lactação no verão.

\section{MATERIAL E MÉTODOS}

O experimento foi realizado em uma granja comercial de suínos, localizada na latitude $18^{\circ} 17^{\prime} 00^{\prime \prime} \mathrm{S}$, longitude $46^{\circ} 59^{\prime} 36^{\prime \prime} \mathrm{N}$ e altitude média de 972 metros, durante os meses de janeiro a março de 2010. O clima da região é caracterizado como mesotérmico Cwa (tropical de altitude, quente/úmido no verão e frio/seco no inverno) pela classificação climática de Köppen. As porcas foram alojadas em galpão com orientação Leste-Oeste, cobertura em telhas de barro, com presença de lanternim, beiral de $0,8 \mathrm{~m}$, sem forro abaixo do telhado, com janelas para controle da ventilação natural e mureta lateral de alvenaria de $1,0 \mathrm{~m}$. O galpão possuía dimensões de 10,0 x 36,0m e pé-direito de 3,0m, com três salas e duas fileiras de 12 baias de 1,7 x $3,4 \mathrm{~m}$ em cada sala. Cada baia tinha uma gaiola para as fêmeas com dimensão de 0,6 x 2,0m e, nas laterais, um espaço exclusivo para os leitões. Foram utilizadas 72 fêmeas suínas da genética DanBred $^{\circledR}$, distribuídas uniformemente nas ordens de parto de primeira a quinta entre os tratamentos (média de ordem de parto igual a 2,9 por tratamento).

O delineamento experimental foi blocos ao acaso com quatro tratamentos em disposição fatorial 2 x 2 e 18 repetições por tratamento. Os fatores foram dois níveis de balanço eletrolítico (BE: $\mathrm{BE}$ e BEA: balanço eletrolítico alto) e dois níveis de sistemas de ventilação (SVN: sistema de ventilação natural e SRE: sistema de resfriamento evaporativo). Os blocos foram oito datas de desmame dos leitões, e a unidade experimental foi a porca com a sua leitegada. Os resultados foram submetidos à análise de variância utilizando o software Minitab ${ }^{\circledR} 15.1$ (Minitab, 2005). Os valores médios foram comparados pelo teste de Tukey e considerou-se o nível de significância de $5 \%(\mathrm{P}<0,05)$, exceto para o peso ao desmame, em que foi considerado o nível de significância de $10 \%(\mathrm{P}<0,1)$, devido à importância econômica desse parâmetro nos resultados de desempenho da suinocultura. As porcas responderam de maneira semelhante ( $\mathrm{P}>0,05)$ aos tratamentos (dois níveis de balanço eletrolítico e dois níveis de sistema de ventilação), independentemente da ordem de parto; portanto, a ordem de parto não foi incluída no modelo estatístico. Como não houve interação significativa entre os tratamentos, as médias dos resultados foram separadas por sistema de ventilação e balanço eletrolítico.

Os níveis nutricionais da ração lactação fornecida do parto até a manifestação do estro das matrizes apresentaram variação apenas no balanço eletrolítico (BE): Ração BE: balanço eletrolítico de $175 \mathrm{mEqkg}^{-1}$ e Ração BEA: 
balanço eletrolítico alto de $275 \mathrm{mEqkg}^{-1}$ (Tab. 1). $\mathrm{Na}$ ração $\mathrm{BE}$, o $\mathrm{BE}$ foi obtido pela fórmula comum da ração lactação. Na ração BEA, o BE foi ajustado com a adição de bicarbonato de sódio $\left(\mathrm{NaHCO}_{3}\right)$ e um produto à base de eletrólitos (Sal eletrolítico $\mathrm{BalEq}{ }^{\circledR} \mathrm{NaK}-$ Química Geral do Nordeste S/A - Brasil). A água foi disponibilizada ad libitum. A ração foi fornecida manualmente, iniciando com $2 \mathrm{~kg}$ no dia do parto e acrescido $1 \mathrm{~kg} \mathrm{dia}^{-1}$ até atingir o consumo ad libitum para todos os tratamentos. A ração durante a lactação foi fornecida às 07h30min, 10h30min, 19h00min e 02h00min. E, durante o intervalo desmame-estro, a ração foi fornecida às $07 \mathrm{~h} 30 \mathrm{~min}$.

Tabela 1. Composição centesimal e calculada das rações experimentais usadas para as porcas em lactação

\begin{tabular}{|c|c|c|c|}
\hline Composição centesimal ingredientes (\%) & & Ração BE & Ração BEA \\
\hline Milho moído 8\% & & 51,000 & 51,000 \\
\hline Farelo de soja $45 \%$ & & 27,000 & 27,000 \\
\hline Farelo de trigo & & 6,000 & 6,000 \\
\hline Óleo vegetal & & 6,000 & 6,000 \\
\hline Açúcar & & 5,000 & 5,000 \\
\hline Fosfato bicálcico & & 1,650 & 1,650 \\
\hline Calcário $37 \%$ & & 1,080 & 1,080 \\
\hline Caulim & & 0,680 & 0,068 \\
\hline Sal moído & & 0,500 & ---- \\
\hline Premix vitamínico-mineral $^{1}$ & & 0,400 & 0,400 \\
\hline L-Lisina $98 \%$ & & 0,365 & 0,365 \\
\hline L-Treonina & & 0,180 & 0,180 \\
\hline DL-Metionina $99 \%$ & & 0,135 & 0,135 \\
\hline Antioxidante & & 0,010 & 0,010 \\
\hline $\mathrm{BalEq}^{\circledR} \mathrm{NaK}^{2}$ & & ---- & 0,635 \\
\hline Bicarbonato de sódio $27 \%$ & & ---- & 0,477 \\
\hline Total & & 100,000 & 100,000 \\
\hline \multicolumn{4}{|l|}{ Composição calculada na matéria natural } \\
\hline Energia Metabolizável Suínos & $\mathrm{Kcal} \mathrm{kg}^{-1}$ & $3.391,54$ & $3.391,54$ \\
\hline Proteína bruta & $\%$ & 17,764 & 17,764 \\
\hline Fibra bruta & $\%$ & 3,411 & 3,411 \\
\hline Extrato etéreo & $\%$ & 8,575 & 8,575 \\
\hline Matéria mineral & $\%$ & 5,723 & 5,243 \\
\hline Cálcio & $\%$ & 0,929 & 0,929 \\
\hline Fósforo total & $\%$ & 0,647 & 0,647 \\
\hline Lisina digestível & $\%$ & 1,068 & 1,068 \\
\hline Sódio & $\%$ & 0,222 & 0,286 \\
\hline Cloro & $\%$ & 0,411 & 0,233 \\
\hline Potássio & $\%$ & 0,762 & 0,850 \\
\hline Balanço Eletrolítico $(\mathrm{BE})^{3}$ & $\mathrm{mEqkg}^{-1}$ & 175,730 & 275,970 \\
\hline
\end{tabular}

${ }^{1}$ Contém em 1,0kg de ração: vit. A: 10.000 UI; vit. $\mathrm{D}_{3}$ : $2.000 \mathrm{UI}$; vit. E: 30mg; vit. $\mathrm{K}_{3}$ : $3 \mathrm{mg}$; vit. $\mathrm{B}_{1}$ : 2mg; vit. $\mathrm{B}_{2}$ : $4 \mathrm{mg}$; vit. $\mathrm{B}_{6}: 3 \mathrm{mg}$; vit. $\mathrm{B}_{12}: 20 \mathrm{mcg}$; colina: $1,4 \mathrm{~g}$; niacina: $25 \mathrm{mg}$; ácido pantotênico: $14 \mathrm{mg}$; ácido fólico: $1,6 \mathrm{mg}$; biotina: 0,4mg; antioxidante: $100 \mathrm{mg}$; ferro: $80 \mathrm{mg}$; manganês: $70 \mathrm{mg}$; zinco: $150 \mathrm{mg}$; cobre: 15mg; iodo: $1 \mathrm{mg}$ e selênio: 0,3mg. ${ }^{2} \mathrm{BalEq}{ }^{\circledR} \mathrm{NaK}$ : sal eletrolítico - mínimo de $21 \%$ de sódio e $20,5 \%$ de potássio e máximo de $19 \%$ de cloro. ${ }^{3}$ Balanço Eletrolítico da ração calculado conforme Patience (1990): $\mathrm{BE}=(\mathrm{Na} / 23+\mathrm{K} / 39-\mathrm{Cl} / 35,5) \times 10000$, com base nos valores das matérias-primas especificados por Rostagno (2005).

As porcas foram submetidas a dois sistemas de ventilação: Sistema de ventilação natural: SVN e Sistema de resfriamento evaporativo: SRE. O SRE foi instalado na extremidade do galpão da maternidade, composto basicamente por painéis evaporativos, ventilador do tipo axial, reservatório de água e sistema interno de circulação e distribuição de água, tudo contido num gabinete metálico, de onde partia um sistema de dutos com saídas individuais para as gaiolas das fêmeas. As aberturas nos dutos eram alternadas, de modo que, dentro de cada sala da 
maternidade avaliada, metade das porcas recebeu o SRE e metade das porcas recebeu o SVN, assim as condições ambientais eram mantidas iguais para todas as porcas alojadas, sendo que metade delas recebia o sistema de ventilação SVN e a outra metade, o sistema de ventilação SRE. O acionamento do sistema de resfriamento evaporativo era ativado quando a temperatura ambiente (TA) atingia $23^{\circ} \mathrm{C}$.

Para aquisição dos dados de TA e UR foi instalado um data logger da marca $\mathrm{HOBO}^{\circledR} \mathrm{em}$ cada sala do galpão de lactação, e os dados foram coletados a cada 60 minutos. A velocidade do ar (VA), TA e UR foram registradas usando um Higro-Termo-Anemômetro (HTA 4300 Marca Pacer, USA), no período da manhã (entre 07h00min e 09h00min) e no período da tarde (entre $15 \mathrm{~h} 00 \mathrm{~min}$ e $17 \mathrm{~h} 00 \mathrm{~min}$ ), diariamente, durante 30s, na região da cabeça da porca em duas posições, deitada e em pé. $O$ índice de conforto térmico foi calculado com base no índice de temperatura e umidade (ITU), de acordo com a equação de Roller e Goldman (1969), citados por Panagakis e Axaopoulos (2006): $\quad I T U=0,45 T B U+1,35 T B S+32$, onde: $\mathrm{TBU}=$ temperatura de bulbo úmido $\left({ }^{\circ} \mathrm{C}\right)$ e TBS $=$ temperatura de bulbo seco $\left({ }^{\circ} \mathrm{C}\right)$.

Os parâmetros de frequência respiratória (FR), temperatura retal (TR) e temperatura superficial (TS) foram registrados diariamente durante a fase lactação, nos períodos da manhã (entre 07h00min e 09h00min) e da tarde (entre 15h00min e 17h00min). A FR foi monitorada visualmente através do número de movimentos do flanco durante um minuto (movimentos por minuto: mpm). A TR foi aferida por meio de termômetro clínico veterinário introduzido no reto das porcas, durante um minuto. A TS foi registrada diariamente com a câmera termográfica infravermelha testo ${ }^{\circledR} 880$, e as imagens termográficas foram processadas pelo software testo IRSoft ${ }^{\circledR}$.

Registraram-se o consumo diário de ração, a condição corporal (peso e espessura de toucinho) e o intervalo desmame-estro das fêmeas suínas, a mortalidade dos leitões na maternidade e o peso dos leitões ao desmame. A quantidade de ração fornecida durante o período do experimento foi pesada diariamente, e as sobras nos comedouros foram colhidas e pesadas para determinação do consumo diário. As porcas foram pesadas antes do parto (peso inicial, em que foi descontado o peso da placenta e dos leitões nascidos) e ao desmame (peso final). A espessura de toucinho também foi mensurada antes do parto (espessura de toucinho inicial) e ao desmame (espessura de toucinho final), com aparelho de ultrassom PigLog- $105^{\circledR}$, na posição $\mathrm{P}_{2}$ (demarcado a $4 \mathrm{~cm}$ da linha dorsal e a $6,5 \mathrm{~cm}$ da última costela na direção cranial). A observação do estro (em dias) teve início a partir do primeiro dia pós-desmame e foi realizada no período da manhã (entre 08h00min e 10h00min) e no período da tarde (entre $15 \mathrm{~h} 00 \mathrm{~min}$ e $17 \mathrm{~h} 00 \mathrm{~min}$ ). A mortalidade dos leitões foi registrada diariamente na maternidade. Os leitões foram pesados individualmente ao nascimento e uniformizados durante o período de até três dias após o parto, permanecendo próximo de 12 leitões por fêmea. O desmame ocorreu, em média, aos 21 dias de idade, quando os leitões foram novamente pesados.

Este trabalho foi aprovado pela Comissão de Ética no Uso de Animais - CEUA/Unicamp sob o protocolo $n^{\circ} 2036-1$.

\section{RESULTADOS E DISCUSSÃO}

Os valores médios encontrados para TA e UR foram de $24,8^{\circ} \mathrm{C}$ e $64,5 \%$, respectivamente, sendo obtidos valores diários de $31,6^{\circ} \mathrm{C}$ para a temperatura ambiente máxima e de $19,8^{\circ} \mathrm{C}$ para a temperatura ambiente mínima. Considerando-se a zona de conforto térmico para porcas em lactação entre $12^{\circ} \mathrm{C}$ e $22^{\circ} \mathrm{C}$ (Black et al., 1993), é possível inferir que as porcas foram submetidas a estresse calórico. $\mathrm{Na}$ avaliação do ambiente térmico de porcas em lactação, Malmkvist et al. (2012) observaram aumento na FR, TR e TS quando TA na sala da maternidade aumentou de $15^{\circ} \mathrm{C}$ para $25^{\circ} \mathrm{C}$.

Pode-se observar o efeito do SRE na redução da TA na região da cabeça da porca, principalmente no período da tarde (Tab. 2), quando havia maior diferença $(\mathrm{P}<0,05)$ entre a TA (mais elevada) e a UR (mais baixa). No SRE, a VA na região da cabeça da porca apresentou o valor médio de $7,2 \mathrm{~m} \mathrm{~s}^{-1}$ na posição em pé e $3,2 \mathrm{~m} \mathrm{~s}^{-1}$ na posição deitada. E, no SVN, a VA na região da cabeça da porca foi de $0,1 \mathrm{~m} \mathrm{~s}^{-1}$. Altas taxas de ventilação são necessárias para produzir velocidade do ar, que pode ter efeito de resfriamento durante períodos de TA elevada, especialmente quando a 
pele do suíno está úmida e ocorre a evaporação da água (Banhazi et al., 2008). Os valores calculados do ITU para o SVN estão muito próximos do limite superior da zona Normal de manhã $(<74)$ e da zona Perigo $(79$ - 83) à tarde (Sampaio et al., 2004).

Tabela 2. Valores médios de UR (\%), TA $\left({ }^{\circ} \mathrm{C}\right.$, TBS e TBU), ITU e classificação da zona de conforto de acordo com o sistema de ventilação (SVN ou SRE), nos períodos da manhã e da tarde

\begin{tabular}{clcc}
\hline & Parâmetros & SVN $^{1}$ & SRE $^{2}$ \\
\hline \multirow{4}{*}{ Manhã } & UR & $77,3 \mathrm{a}$ & $85,5 \mathrm{~b}$ \\
& TBS & $24,0 \mathrm{a}$ & $22,2 \mathrm{~b}$ \\
& TBU & $21,0 \mathrm{a}$ & $20,2 \mathrm{~b}$ \\
& ITU & $73,8 \mathrm{a}$ & $71,0 \mathrm{~b}$ \\
& Zona de conforto & Normal & Normal \\
\hline \multirow{3}{*}{ Tarde } & UR & $62,4 \mathrm{a}$ & $73,6 \mathrm{~b}$ \\
& TBS & $28,4 \mathrm{a}$ & $26,0 \mathrm{~b}$ \\
& TBU & $22,6 \mathrm{a}$ & $22,3 \mathrm{~b}$ \\
& ITU & $80,6 \mathrm{a}$ & $77,2 \mathrm{~b}$ \\
& Zona de conforto & Perigo & Alerta \\
\hline
\end{tabular}

Letras distintas na mesma linha indicam diferença significativa $(\mathrm{P}<0,05)$.

${ }^{1} \mathrm{SVN}$ : para o cálculo do ITU, foram utilizados os valores registrados do ambiente interno para UR e TBS.

${ }^{2}$ SRE: para o cálculo do ITU, foram utilizados os valores médios de UR e TBS obtidos na região da cabeça da porca nas posições em pé e deitada.

A dieta com balanço eletrolítico alto não promoveu diferença significativa $(\mathrm{P}>0,05)$ nos parâmetros de termorregulação (Tab. 3). No entanto, o SRE proporcionou redução significativa $(\mathrm{P}<0,05)$ nos valores de $\mathrm{FR}$, resultado este que corrobora os obtidos por Romanini et al. (2008), e de TS, nos períodos da manhã e da tarde, e da TR mensuradas no período da tarde. Como os suínos não possuem mecanismo eficaz de perda de calor pela evaporação na pele, dependem da eficácia da FR (Black et al., 1993; Thuy, 2005). A redução na FR no SRE foi de $22 \%(\mathrm{P}<0,05)$ no período da manhã e de $29,5 \%(\mathrm{P}<0,05)$ no período da tarde. Todavia, neste estudo, a FR apresentou-se acima dos valores referenciais entre 15 e $22 \mathrm{mpm}$ relatados pelo National..., (2011).

Tabela 3. Parâmetros de termorregulação das fêmeas suínas de acordo com o sistema de ventilação (SRE ou SVN) e o balanço eletrolítico da dieta (BEA ou BE)

\begin{tabular}{|c|c|c|c|c|c|c|}
\hline \multirow[b]{2}{*}{ Variável } & \multicolumn{3}{|c|}{ Sistema de ventilação } & \multicolumn{3}{|c|}{ Balanço eletrolítico } \\
\hline & & $\begin{array}{l}\text { Média } \pm \text { erro } \\
\text { padrão }\end{array}$ & $\begin{array}{l}\text { P- } \\
\text { Valor }\end{array}$ & & $\begin{array}{c}\text { Média } \pm \text { erro } \\
\text { padrão }\end{array}$ & $\begin{array}{l}\mathrm{P}- \\
\text { Valor }\end{array}$ \\
\hline \multirow{2}{*}{$\begin{array}{l}\text { Frequência respiratória } \\
\text { manhã (mpm) }\end{array}$} & SRE & $37,92 \pm 1,58$ & \multirow{2}{*}{$*$} & BEA & $41,83 \pm 1,58$ & \multirow{2}{*}{ NS } \\
\hline & SVN & $46,25 \pm 1,57$ & & $\mathrm{BE}$ & $42,33 \pm 1,59$ & \\
\hline \multirow{2}{*}{$\begin{array}{l}\text { Frequência respiratória à } \\
\text { tarde }(\mathrm{mpm})\end{array}$} & SRE & $56,42 \pm 2,07$ & \multirow{2}{*}{$*$} & BEA & $68,22 \pm 2,24$ & \multirow{2}{*}{ NS } \\
\hline & SVN & $80,09 \pm 2,57$ & & $\mathrm{BE}$ & $68,29 \pm 2,23$ & \\
\hline Temperatura retal manhã & SRE & $38,53 \pm 0,05$ & \multirow{2}{*}{ NS } & BEA & $38,56 \pm 0,05$ & \multirow{2}{*}{ NS } \\
\hline$\left({ }^{\circ} \mathrm{C}\right)^{*}$ & SVN & $38,54 \pm 0,05$ & & $\mathrm{BE}$ & $38,52 \pm 0,05$ & \\
\hline Temperatura retal à tarde & SRE & $39,12 \pm 0,06$ & \multirow{2}{*}{$*$} & BEA & $39,25 \pm 0,06$ & \multirow{2}{*}{ NS } \\
\hline$\left({ }^{\circ} \mathrm{C}\right)^{1}$ & SVN & $39,41 \pm 0,07$ & & $\mathrm{BE}$ & $39,29 \pm 0,08$ & \\
\hline Temperatura superficial & SRE & $33,04 \pm 0,08$ & \multirow{2}{*}{$*$} & BEA & $33,24 \pm 0,08$ & \multirow{2}{*}{ NS } \\
\hline manhã $\left({ }^{\circ} \mathrm{C}\right)$ & SVN & $33,46 \pm 0,08$ & & $\mathrm{BE}$ & $33,26 \pm 0,08$ & \\
\hline Temperatura superficial à & SRE & $35,61 \pm 0,09$ & \multirow{2}{*}{$*$} & BEA & $35,90 \pm 0,10$ & \multirow{2}{*}{ NS } \\
\hline $\operatorname{tarde}\left({ }^{\circ} \mathrm{C}\right)$ & SVN & $36,13 \pm 0,08$ & & $\mathrm{BE}$ & $35,83 \pm 0,10$ & \\
\hline
\end{tabular}

$\mathrm{P}-$ Valor $=*$ : significativo $(\mathrm{P}<0,05) ; \mathrm{P}-$ Valor $=$ NS: não significativo.

A TR elevada no período da tarde indica que os suínos podem ter esgotado sua capacidade de termorregulação e que os esforços de aumento na
FR, diminuição na produção de calor por menor atividade, mudança na postura corporal e redução no consumo de ração não foram 
suficientes para perder calor (Thuy, 2005), confirmando que a resposta da temperatura retal à mudança no conteúdo de calor corporal é relativamente lenta e tardia (Silva, 2006). A redução na TS com a utilização do SRE está de acordo com as observações de Jiang et al. (2004), em que o aumento nas perdas de calor por evaporação e convecção resultou em menor $(\mathrm{P}<0,05) \quad$ TS. Além disso, a TS refletiu diretamente a resposta do suíno à TA (Thuy, 2005). E, como nos estudos de Moura et al.
(2011) e Brown-Brandl et al. (2012), o uso da termografia infravermelha permitiu precisão na determinação da TS e sua associação com a termorregulação.

Pode ser observado que a FR (Fig. 1), a TS (Fig. 2) e a TR (Fig. 3) das porcas apresentaram valores mais elevados no período da tarde em relação ao período da manhã e os efeitos do sistema de ventilação nessas variáveis.

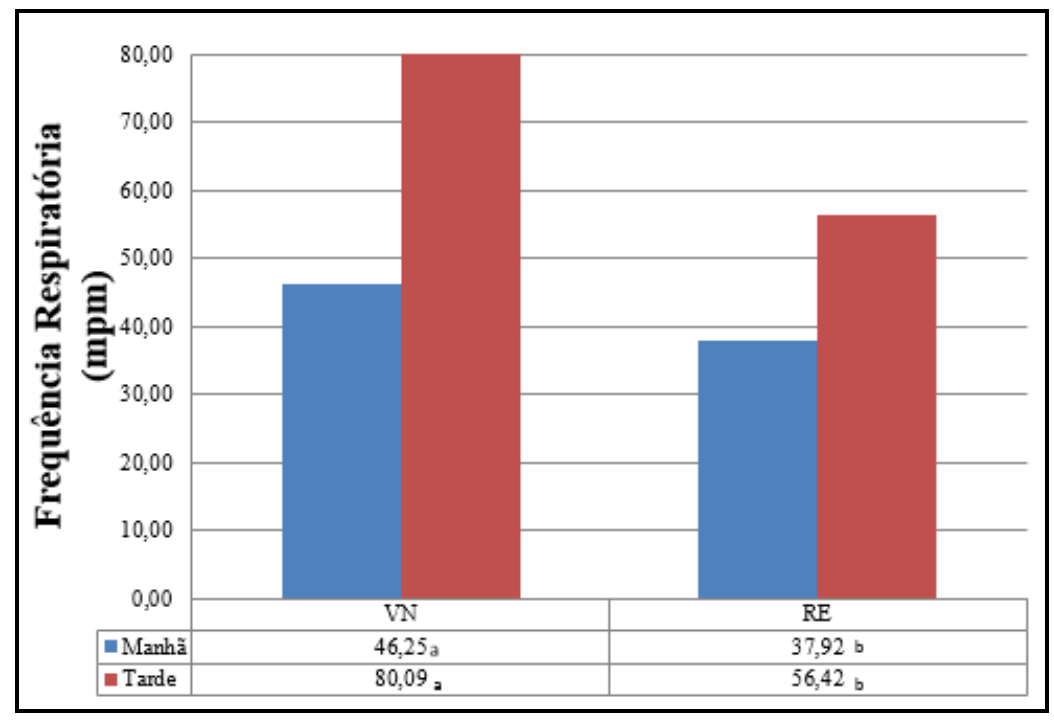

a, b - Letras distintas na mesma linha indicam diferença significativa $(\mathrm{P}<0,05)$.

Figura 1. Valores médios da frequência respiratória das porcas durante os períodos da manhã e da tarde, no sistema de ventilação natural (VN) e no sistema de resfriamento evaporativo (RE).

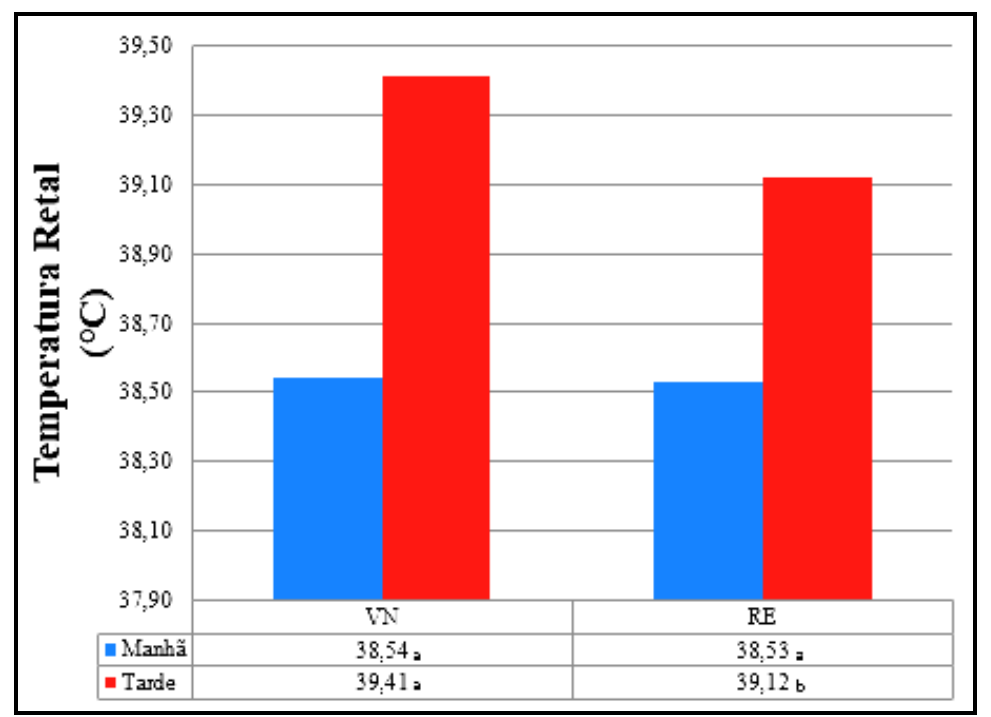

a, b - Letras distintas na mesma linha indicam diferença significativa $(\mathrm{P}<0,05)$.

Figura 2. Valores médios da temperatura retal das porcas durante os períodos da manhã e da tarde, no sistema de ventilação natural (VN) e no sistema de resfriamento evaporativo (RE). 


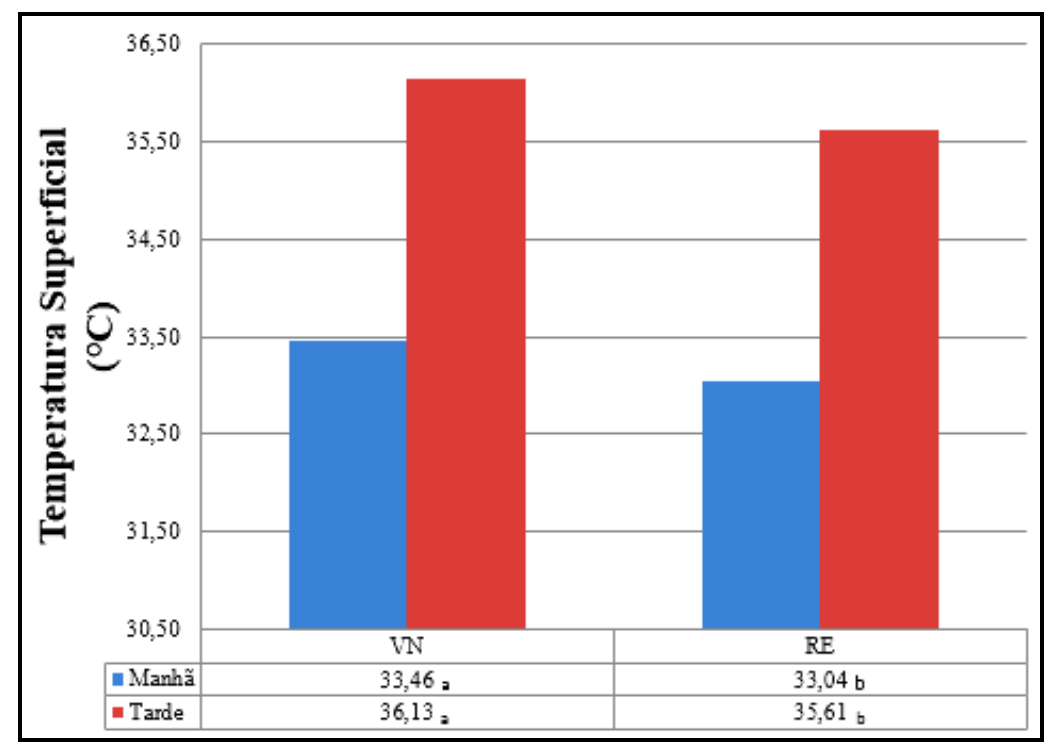

a, b - Letras distintas na mesma linha indicam diferença significativa $(\mathrm{P}<0,05)$.

Figura 3. Valores médios da temperatura superficial das porcas durante os períodos da manhã e da tarde, no sistema de ventilação natural (VN) e no sistema de resfriamento evaporativo (RE).

Não foram observados efeitos significativos $(\mathrm{P}>0,05)$ do sistema de ventilação e do balanço eletrolítico da dieta sobre as variáveis relacionadas ao consumo de ração, peso corporal, espessura de toucinho e intervalo desmame-estro das porcas (Tab. 4). Resultados estes encontrados também por Dove e Haydon (1994) em relação ao balanço eletrolítico da dieta. A ração lactação com menor incremento calórico (Brown-Brandl et al., 2004), através de alta inclusão de óleo vegetal e aminoácidos e nível adequado de proteína bruta, certamente contribuiu para o consumo suficiente dos nutrientes, mesmo no verão. Desse modo, tanto o balanço eletrolítico alto como o sistema de resfriamento evaporativo não apresentaram efeito significativo nessas variáveis.

Segundo Kemp et al. (2011), dietas ricas em gordura podem ser benéficas em climas quentes, considerando-se que a produção de calor é menor quando a gordura é usada para produção de leite em comparação a carboidratos. $\mathrm{Na}$ avaliação realizada por Teixeira (2010), o balanço eletrolítico das dietas de lactação $(150,199,247$ e $\left.297 \mathrm{mEqkg}^{-1}\right)$ também não interferiu $(P>0,05)$ no consumo de ração das matrizes durante a lactação sob condições de temperatura ambiente acima de $29^{\circ} \mathrm{C}$. O autor sugeriu que o suíno apresenta uma rápida capacidade de compensação renal aos desequilíbrios eletrolíticos em granjas com manejos adequados e elevado padrão sanitário. Black et al. (1993) relataram que aumentar a perda de calor da porca, particularmente através da evaporação da pele, apresenta efeito positivo maior sobre o desempenho do que a modificação da dieta.

Tendo em vista o consumo médio de ração de $6,96 \mathrm{~kg}$ por dia, as porcas deste experimento, em geral, não entraram em catabolismo, ao contrário, apresentaram ganho de peso médio de $4,0 \mathrm{~kg}$ durante os 20 dias de lactação. Estes resultados confirmam as recomendações de Bortolozzo et al. (2011) no sentido de reduzir a perda de peso na fase lactacional melhorando o conforto ambiental e o consumo voluntário de ração. Utilizando níveis de balanço eletrolítico das rações gestação e lactação, entre $117 \mathrm{e}$ $297 \mathrm{mEqkg}^{-1}$ de ração, Teixeira (2010) também não encontrou resposta para a variável perda de peso da matriz durante a lactação. Não foi observada diferença significativa $(P>0,05)$ entre os tratamentos para o intervalo desmame-estro, provavelmente em razão do bom estado metabólico geral das porcas após o desmame. 
Tabela 4. Parâmetros de desempenho das porcas e dos leitões de acordo com o sistema de ventilação (SRE ou SVN) e o balanço eletrolítico da dieta (BEA ou BE)

\begin{tabular}{|c|c|c|c|c|c|c|}
\hline \multirow[b]{2}{*}{ Variável } & \multicolumn{3}{|c|}{ Sistema de ventilação } & \multicolumn{3}{|c|}{ Balanço eletrolítico } \\
\hline & & $\begin{array}{c}\text { Média } \pm \text { erro } \\
\text { padrão }\end{array}$ & $\begin{array}{c}\text { P- } \\
\text { Valor }\end{array}$ & & $\begin{array}{c}\text { Média } \pm \text { erro } \\
\text { padrão }\end{array}$ & $\begin{array}{l}\text { P- } \\
\text { Valor }\end{array}$ \\
\hline \multirow{2}{*}{$\begin{array}{l}\text { Consumo de ração diário } \\
(\mathrm{kg})\end{array}$} & SRE & $7,131 \pm 0,147$ & \multirow{2}{*}{ NS } & BEA & $6,923 \pm 0,154$ & \multirow{2}{*}{ NS } \\
\hline & SVN & $6,787 \pm 0,138$ & & $\mathrm{BE}$ & $6,995 \pm 0,136$ & \\
\hline Peso inicial & SRE & $234,41 \pm 4,88$ & \multirow{2}{*}{ NS } & BEA & $235,86 \pm 5,59$ & \multirow{2}{*}{ NS } \\
\hline$(\mathrm{kg})^{1}$ & SVN & $238,31 \pm 5,47$ & & $\mathrm{BE}$ & $236,86 \pm 4,76$ & \\
\hline Peso final & SRE & $240,71 \pm 5,29$ & \multirow{2}{*}{ NS } & BEA & $240,13 \pm 6,10$ & \multirow{2}{*}{ NS } \\
\hline$(\mathrm{kg})$ & SVN & $240,10 \pm 6,04$ & & $\mathrm{BE}$ & $240,68 \pm 5,10$ & \\
\hline Espessura de toucinho inicial & SRE & $19,17 \pm 0,79$ & \multirow{2}{*}{ NS } & BEA & $18,69 \pm 0,68$ & \multirow{2}{*}{ NS } \\
\hline$(\mathrm{mm})$ & SVN & $18,78 \pm 0,66$ & & $\mathrm{BE}$ & $19,25 \pm 0,78$ & \\
\hline Espessura de toucinho final & SRE & $18,86 \pm 0,76$ & \multirow{2}{*}{ NS } & BEA & $17,97 \pm 0,67$ & \multirow{2}{*}{ NS } \\
\hline$(\mathrm{mm})$ & SVN & $18,06 \pm 0,64$ & & $\mathrm{BE}$ & $18,94 \pm 0,73$ & \\
\hline Intervalo desmame-estro & SRE & $4,9 \pm 0,20$ & \multirow{2}{*}{ NS } & BEA & $4,8 \pm 0,20$ & \multirow{2}{*}{ NS } \\
\hline$($ dias $)$ & SVN & $4,7 \pm 0,20$ & & $\mathrm{BE}$ & $4,9 \pm 0,20$ & \\
\hline Número de leitões & SRE & $11,97 \pm 0,16$ & \multirow{3}{*}{ NS } & BEA & $12,07 \pm 0,15$ & \multirow{3}{*}{ NS } \\
\hline Uniformizados & SVN & $12,12 \pm 0,15$ & & $\mathrm{BE}$ & $12,02 \pm 0,14$ & \\
\hline Mortalidade & SRE & $0,84 \pm 0,27$ & & BEA & $1,15 \pm 0,27$ & \\
\hline$(\%)$ & SVN & $0,84 \pm 0,28$ & \multirow[t]{2}{*}{ NS } & $\mathrm{BE}$ & $0,54 \pm 0,26$ & \multirow[t]{2}{*}{ NS } \\
\hline Número de leitões & SRE & $11,19 \pm 0,15$ & & BEA & $11,38 \pm 0,16$ & \\
\hline Desmamados & SVN & $11,64 \pm 0,16$ & \multirow[t]{2}{*}{ NS } & $\mathrm{BE}$ & $11.45 \pm 0,17$ & \multirow[t]{2}{*}{ NS } \\
\hline Peso médio do leitão ao & SRE & $1,511 \pm 0,044$ & & BEA & $1,504 \pm 0,041$ & \\
\hline $\operatorname{nascer}(\mathrm{Kg})$ & SVN & $1,496 \pm 0,048$ & \multirow[t]{2}{*}{ NS } & $\mathrm{BE}$ & $1,535 \pm 0,046$ & \multirow[t]{2}{*}{ NS } \\
\hline Peso médio do leitão ao & SRE & $6,857 \pm 0,162$ & & BEA & $6,702 \pm 0,156$ & \\
\hline desmame $(\mathrm{Kg})$ & SVN & $6,627 \pm 0,125$ & $* *$ & $\mathrm{BE}$ & $6,782 \pm 0,134$ & NS \\
\hline
\end{tabular}

$\mathrm{P}-$ Valor $=* *$ : significativo $(\mathrm{P}<0,1) ; \mathrm{P}-$ Valor $=\mathrm{NS}$ : não significativo.

${ }^{1}$ Peso inicial das fêmeas sem placenta e leitões.

Considerando-se o alto status sanitário da granja, não houve efeito significativo $(\mathrm{P}>0,05)$ do sistema de ventilação ou do balanço eletrolítico da dieta sobre a mortalidade, e o número de leitões manteve-se em média 11,4 leitões desmamados por fêmea. Kiefer et al. (2012) também não encontraram diferença significativa no número de leitões desmamados ou mortalidade ao desmame para porcas submetidas ao SRE na maternidade. Teixeira et al. (2004) igualmente obtiveram maior peso dos leitões ao desmame com o SRE. Neste trabalho, houve diferença significativa ao nível de $\mathrm{P}<0,10$ para essa variável. O maior peso ao desmame $(6,857 \mathrm{~kg}$ para o SRE e $6,627 \mathrm{~kg}$ para o SVN) pode estar relacionado à maior perda de calor para o ambiente no SRE e à boa produção de leite das fêmeas em consequência ao consumo de ração suficiente para atender às exigências nutricionais. Black et al. (1993) descreveram que a produção de leite das fêmeas em condições de temperatura elevada pode ser mantida pela redução na produção de calor ou aumento na perda de calor para o ambiente. O SRE foi direcionado à região da cabeça da porca, diretamente ao hipotálamo, ou seja, influenciou na fêmea e não no microclima do galpão (Tolon e Nääs, 2005), não ocasionando, portanto, qualquer problema no desenvolvimento dos leitões.

\section{CONCLUSÕES}

A utilização do sistema de resfriamento evaporativo contribuiu para reduzir os efeitos do calor sobre as variáveis relacionadas à termorregulação das matrizes suínas em lactação durante o verão e proporcionou aumento no peso dos leitões ao desmame. Em dieta com níveis nutricionais específicos para climas quentes, o balanço eletrolítico alto não minimizou os efeitos do estresse calórico.

\section{AGRADECIMENTOS}

À empresa Agroceres Multimix e à Fundação de Amparo à Pesquisa - FAPESP, pelo financiamento para a realização da pesquisa. 


\section{REFERÊNCIAS}

AARNINK, A.J.A.; SCHRAMA, J.W.; HEETKAMP, M.J.W. et al. Temperature and body weight affect fouling of pig pens. J. Anim. Sci., v.84, p.2224-2231, 2006.

BANHAZI, T.M.; SEEDORF, J.; RUTLEY, D.L.; PITCHFORD W.S. Identification of risk factors for sub-optimal housing conditions in Australian piggeries: Part 3. Environmental parameters. J. Agricultural Safety and Health, v.14, p.41-52, 2008 a.

BLACK, J.L.; MULLAN, B.P.; LORSCHY, M.L.; GILES, L.R. Lactation in the sow during heat stress. Livest. Prod. Sci., v.35, p.153-170, 1993.

BLOEMHOF, S.; VAN DER WAAIJ, E.H.; MERKS, J.W.M.; KNOL, E.F. Sow line differences in heat stress tolerance expressed in reproductive performance traits. J. of Anim. Scie., v.86, p.3330-3337, 2008.

BORTOLOZZO, F.P.; GAGGINI, T.S.; WENTZ, I. Infertilidade sazonal no suíno: caracterização e consequências durante a fase gestacional. In: SIMPÓSIO INTERNACIONAL DE SUINOCULTURA. Produção, Reprodução e Sanidade Suína. 6., 2011, Porto Alegre. Anais... Porto Alegre: [s.n.] 2011. p.117-131.

BROWN-BRANDL, T.M.; EIGENBERG, R.A.; PURSWELL, J.L. Determining heat tolerance in finishing pigs using thermal imaging. In: INTERNATIONAL LIVESTOCK ENVIRONMENT SYMPOSIUM, 9., 2012, Valencia. Proceedings... Valencia: ASABE, 8p., 2012.

BROWN-BRANDL, T.M.; NIENABER, J.A.; XIN, H.; GATES, R.S. A literature review of swine heat production. Transaction of the $A S A B E$, v.47, p.259-270, 2004.

DOVE, C.R.; HAYDON, K.D. The effect of various diet nutrient densities and electrolyte balances on sow and litter performance during two seasons of the year. J. Anim. Sci., v.72, p.1101-1106, 1994.

JIANG, M.; GEBREMEDHIN, K.G.; ALBRIGHT, L.D. Numerical Simulation of Coupled Heat and Mass Transfer through the Hair Coat. In: ASAE/CSAE Annual International Meeting, 2004, Ontario. Proceedings... Ontario: ASAE, 2004.
KEMP, B.; WIENTJES, A.J.; LEEUWEN, V. et al Key factors to improve production and longevity of primiparous sows. In: SIMPÓSIO INTERNACIONAL DE SUINOCULTURA. PRODUÇÃO, REPRODUÇÃO E SANIDADE SUÍNA, 6., 2011, Porto Alegre. Anais... Porto Alegre, p.13-22, 2011.

KIEFER, C.; MARTINS, L.P.; FANTINI, C.C. Evaporative cooling for lactating sows under high ambient temperature. Rev. Bras. Zootec., v.41, p.1180-1185, 2012.

LESSKIU, P.E.; GONÇALVES, M.A.D.; BRANDT, G. et al. Descarte de fêmeas jovens: racionalização das políticas de descarte e seus impactos sobre a produtividade do plantel. In: SIMPÓSIO INTERNACIONAL DE SUINOCULTURA. PRODUÇÃO, REPRODUÇÃO E SANIDADE SUÍNA. 6., 2011, Porto Alegre. Anais... Porto Alegre: 2011. p.139-161.

LIMA, A.L.; OLIVEIRA, R.F.M.; DONZELE, J.L.; FERNANDES, H.C. et al. Resfriamento do piso da maternidade para porcas em lactação no verão. Rev. Bras. Zootec., v.40, p.804-811, 2011.

MALMKVIST, J.; PEDERSEN, L.J.; KAMMERSGAARD, T.S.; JORGENSEN, E. Influence of thermal environment on sows around farrowing and during the lactation period. J. Anim. Sci., v.90, p.3186-3199, 2012.

MARTIN, W.R. Effects of heat stress on thermoregulation, reproduction and performance of different parity sows. 2012. 154f. Thesis (Master of Science) - Faculty of the Graduate School University of Missouri, Missouri, 2012.

MINITAB. Versão 15.1.0.0. Inc. 2005.

MOURA, D.J.; MAIA, A.P.A.; VERCELLINO, R.A. et al. Uso da termografia infravermelha na análise da termorregulação de cavalo em treinamento. Eng. Agrícola, v.31, p.23-32, 2011.

NATIONAL Pork Board. Provide proper swine care to improve swine well-being: ventilation. In: SWINE CARE HANDBOOK. Des Moines: Iowa, 2011. p.98-99.

PANAGAKIS, P.; AXAOPOULOS, P. Simulation comparison of fogging strategies based on growing swine apparent heat-stress indices. In: AGENG'06, AGRICULTURAL ENGINEERING FOR A BETTER WORLD, 2006, Bonn. Proceedings... Germany: Bonn, 6p., 2006. 
PATIENCE, J.F. A review of the role of acidbase balance in amino acid nutrition. J. Anim. Sci., v.68, p.398-408, 1990.

ROMANINI, C.E.B.; TOLON, Y.B.; NÄÄS, I.A.; MOURA, D.J. Physiological and productive responses of environmental control on housed sows. Science Agricul., v.65, p.335-339, 2008.

ROSTAGNO, H.S. Tabelas Brasileiras para Aves e Suínos: Composição de Alimentos e Exigências Nutricionais. Viçosa: UFVDepartamento de Zootecnia, 252p., 2005.

SAMPAIO, C.A.P.; CRISTIANI, J.; DUBIELA, J.A. et al. Avaliação do ambiente térmico em instalação para crescimento e terminação de suínos utilizando os índices de conforto térmico nas condições tropicais. Cienc. Rural, v.34, p.19, 2004.

SAVARIS, V.D.L. Estudo do balanço eletrolítico $e$ da proteína bruta da ração para suínos em crescimento em condições de alta temperatura. 2008. 59f. Dissertação (Mestrado em Produção Animal) - Faculdade de Zootecnia, Universidade Estadual do Norte Fluminense Darcy Ribeiro, Rio de Janeiro.
SILVA, B.A.N.; OLIVEIRA, R.F.M.; DONZELE, J.L. et al. Effect of floor cooling on performance of lactating sows during summer. Livestock Sci, v.105, p.176-184, 2006.

TEIXEIRA, H.S. Impacto do balanço eletrolítico sobre parâmetros reprodutivos da fêmea suína. 60f. Dissertação (Mestrado em Ciências Veterinárias) - Setor de Ciências Veterinárias, Universidade Federal do Paraná, 2010.

TEIXEIRA, V.H.; TEIXEIRA, A.S.; LOPES, S.P. Efeito do resfriamento adiabático evaporativo e da ventilação forçada no desempenho de porcas lactantes e suas leitegadas. Eng. na Agricultura, v.12, p.51-56, 2004.

THUY, H.T.T. Heat stress in growing pigs. 2005. 163f. Thesis (Phd in Animal Science) Wageningen Institute of Animal Science, Wageningen University, Wageningen, The Netherlands. 2005.

TOLON, Y.B; NÄÄS, I.A. Avaliação de tipos de ventilação em maternidade de suínos. Eng. Agrícola, v.25, p.565-574, 2005. 